\title{
EFEITO DA FARINHA DE RESÍDUO DE PEIXE E DE FRANGO NO DESEMPENHO E NA COMPOSIÇÃO CORPORAL DE JUVENIS DE TAMBAQUI, Colossoma macropomum (CUVIER, 1818)
}

\author{
Werner Damião Morhy TERRAZAS ${ }^{2}$, Manoel PEREIRA-FILHOํ, Maria Inêz \\ de OLIVEIRA-PEREIRA ${ }^{3}$
}

\begin{abstract}
RESUMO - Este trabalho teve como objetivos testar o efeito de rações com um nível de farinha de resíduo de peixe e um de resíduo de frango, assim como a combinação de dois níveis desses ingredientes sobre o ganho de peso e composição corporal de juvenis de tambaqui, Colossoma macropomum. $\mathrm{O}$ experimento foi realizado em delineamento inteiramente casualizado com quatro tratamentos (rações experimentais) cada um com quatro repetições. As rações experimentais possuiam as seguintes formulações: $\mathrm{R} 1=21 \%$ de farinha de resíduo de peixe; $\mathrm{R} 2=14 \%$ de farinha de resíduo de peixe $+7 \%$ farinha de resíduo de frango; $\mathrm{R} 3=7 \%$ de farinha de resíduo de peixe $+14 \%$ farinha de resíduo de frango e $\mathrm{R} 4=21 \%$ de farinha de resíduo de frango. Em cada um dos 16 tanques, foram estocados 16 peixes distribuídos homogeneamente entre os tratamentos $(\mathrm{p}>0,05)$ com peso médio inicial de $13,9 \pm 0,4 \mathrm{~g}$, por um período de 120 dias. Os resultados para o desempenho e composição corporal dos peixes não apresentaram diferença significativa entre os tratamentos (ANOVA $p>0,05$ ), sendo que esses parâmetros mostraram melhores resultados quando houve a combinação das duas farinhas de resíduo.
\end{abstract}

Palavras-chave: Colossoma macropomum, farinha de resíduo de peixe, farinha de resíduo de frango. Effect of Different Levels of Fish Meal and Chicken By-Product Meal in the Weight Gain and Body Composition of Tambaqui (Colossoma macropomum)

ABSTRACT - This study attempts to verify the effect of fish meal (FM), poultry meal (PM) and a combination of these ingredients on the weight gain and body composition of juvenile tambaqui, Colossoma macropomum. The experiment was conducted in a complete randomized design using four treatments, each one with four replicates. The test diets were as follows: R1 = $21 \% \mathrm{FM} ; \mathrm{R} 2=14 \% \mathrm{FM}+7 \% \mathrm{PM} ; \mathrm{R} 3=7 \% \mathrm{FM}+14 \% \mathrm{PM}$ and $\mathrm{R} 4=21 \% \mathrm{PM}$. Fish were stocked in 16 tanks at a density of 16 fish per tank. Fish initial mean weight was $13.9 \pm 0.4 \mathrm{~g}$. After 120 days, the results showed no significant differences among treatments $(p>0.05)$ although an improvement in the weight gain was observed when FM and PM were used in combination.

Key-words: Colossoma macropomum, fish meal, chicken meal.

\section{INTRODUÇÃO}

A disponibilidade de pescado e de produtos da pesca para o consumo no Brasil é de 5,7. kg/hab/ano, o qual é considerado baixo quando comparado aos países desenvolvidos cuja média é de $26,6 \mathrm{~kg} / \mathrm{hab} / \mathrm{ano}$, e a outros em desenvolvimento com média de 9,2 kg/hab/ano (FAO, 1990). Entretanto, um estudo da pesca artesanal realizado pelo BASA (1995) afirma que a disponibilidade de pescado no Estado do Pará é de 23,0 $\mathrm{kg} / \mathrm{hab} / \mathrm{ano}$, sendo que, nas comunidades pesqueiras, o consumo chega a $72,0 \mathrm{~kg} / \mathrm{hab} / \mathrm{ano}$. Estes números, embora obtidos no estado do

1. Parte da Dissertação de Mestrado do primeiro Autor.

2. Universidade Federal do Pará, Campus Santarém, Pa.

3. INPA. Caixa Postal 478. Manaus, Am. Brasil. 69011-970 
Pará, sugerem que a população amazônica tem o peixe incorporado ao seu hábito alimentar e portanto são consumidores em potencial de pescado proveniente de cultivos racionais.

Deve-se ainda ressaltar que a região amazônica conta com a maior bacia hidrográfica do mundo e com um clima favorável à prática da piscicultura Por estes motivos novas tecnologias precisam ser desenvolvidas, visando melhorar a produtividade na piscicultura e incentivar o produtor a investir no setor aquícola.

Alguns autores verificaram a diminuição no desembarque de tambaqui e de outras espécies de peixes de interesse comercial, nos principais centros de consumo (Junk, 1975; Petrere Jr. ,1978; Merona \& Bittencourt, 1988; Falabella, 1994; Graef, 1995). Essas estatísticas sugerem a existência de depleção dos estoques naturais dessas espécies devido ao crescente esforço de pesca. Devido a esses fatores a piscicultura passou a ser uma solução para a produção de peixe e de acordo com Rodrigues et al. (1996), a produção de pescado cultivado tem crescido de forma significativa nas últimas décadas, alcançando no ano de 1992 cerca de $1 / 4$ da produção de pescado consumido no mundo.

Um dos principais problemas que pode ser enfrentado no fomento à criação racional de peixes na região, é como alimentá-los, tendo em vista que o fator alimentação tem uma participação significativa no custo de produção, representando entre 55 e $70 \%$ do total (Torres,1989).
Andriguetto et al. (1990) sugerem que os alimentos usados na alimentação humana devem ser descartados da alimentação animal com a finalidade de evitar a competição por esses insumos com populações humanas.

As dificuldades na aquisição de ingredientes regionais para a formulação de rações foram citadas por Saint-Paul \& Werder (1977). Com o aumento da piscicultura e com a realização de novas pesquisas, novas matérias-primas para rações foram encontradas, como peixes sem interesse comercial e seus subprodutos, resíduos do abate de frangos (PereiraFilho, 1982); fontes de matéria-prima de origem vegetal como sementes e frutos de áreas alagáveis (Roubach, 1991; Silva, 1997) e a possibilidade de utilização de macrófitas aquáticas direta ou indiretamente na alimentação animal (Junk, 1979; Saint-Paul et al., 1981; Saint-Paul, 1986).

Considerando a importância da redução dos custos de produção de rações, uma alternativa seria a formulação de rações a partir do uso de resíduos agroindustriais, ressalvados os aspectos tóxicos e custos de processamento que os tornem utilizáveis. Sendo assim este trabalho tem por objetivo comparar o efeito de duas fontes protéicas (farinhas de resíduos de peixe e de frango) através do desempenho em crescimento, ganho de peso e composição corporal de alevinos de tambaqui, Colossoma macropomum.

\section{MATERIAL E MÉTODOS}

Os experimentos foram realizados nas instalações da Estação 
de Piscicultura de Santa Rosa no município de Santarém, estado do Pará. O processamento, formulação e análise de resíduos foram feitos no Laboratório de Nutrição de Peixes da Coordenação de Pesquisas em Aquicultura do INPA, em Manaus-AM.

$\mathrm{O}$ experimento foi realizado em delineamento inteiramente casualizado com quatro tratamentos (rações experimentais) cada um com quatro repetições. Foram usados 16 tanques (unidades experimentais), construídos em alvenaria medindo $2 \times 4 \mathrm{~m}$ revestidos em concreto e volume de $9,6 \mathrm{~m}^{3}$, com renovação contínua de água por meio de canais de abastecimento e de drenagem. Os peixes foram distribuídos homogeneamente nos tratamentos $(\mathrm{p}>0,05), 16$ peixes por tanque, totalizando 256 peixes com peso médio inicial de $13,9 \pm 0,4 \mathrm{~g}$, por um período experimental de 120 dias. Os peixes foram alimentados duas vezes ao dia (09:00 e 15:00 h) à razão de 3\% da biomassa de cada tanque, reajustado a cada mês após a pesagem dos peixes.

Todos os peixes de cada unidade experimental foram submetidos a biometrias mensais de peso e comprimento. Antes das biometrias os peixes foram acondicionados em balde com água e anestesiados com EtilenoGlicol-Monofenil-Éter na concentração de $0,03 \%$, com a finalidade de diminuir o estresse dos peixes e facilitar seu manuseio.

O processamento dos resíduos dos peixes foi realizado segundo Beaumaríage (1968) e Freitas \& Gurgel (1976) e os resíduos de frango foram obtidos da Varig Agropecuária, Santarém, Pará.

Foram formuladas quatro rações contendo como ingredientes variáveis, as farinhas de resíduos de peixe e de frango, e como ingredientes fixos quirera de arroz, farelo de soja, farinha de trigo, pré-mistura de vitaminas e de minerais (Tab. 1). Todas as rações fo-

Tabela 1. Composição percentual das rações experimentais $(\mathrm{R} 1=21 \%$ de farinha de resíduo de peixe; $\mathrm{R} 2=14 \%$ de farinha de resíduo de peixe $+7 \%$ farinha de resíduo de frango; $\mathrm{R} 3=7 \%$ de farinha de resíduo de peixe $+14 \%$ farinha de resíduo de frango; $\mathrm{R} 4=21 \%$ de farinha de resíduo de frango).

\begin{tabular}{lcccc}
\hline & \multicolumn{3}{c}{ RAÇÕES } \\
\cline { 2 - 5 } Ingredientes (\%) & R1 & R2 & R3 & R4 \\
\hline Farinha resíduos peixes & 21 & 14 & 7 & 21 \\
Farinha residuos frangos & 0 & 7 & 55 & 55 \\
\hline Quirera, arroz quebrado & 55 & 55 & 18 & 18 \\
Farelo de soja & 18 & 5 & 5 & 5 \\
Farinha de trigo & 5 & 0,5 & 0,5 & 0,5 \\
Pré-mistura vitaminas1 & 0,5 & 0,5 & 0,5 & 0,5 \\
Pré-mistura minerais2 & 0,5 & 100 & 100 & 100 \\
\hline Totais (\%) & 100 & & 5,5 \\
\hline
\end{tabular}

' Rovimix Vitaminas â Lab. Roche S.A. : Vit. A 8,0. $10^{6}$ UI; Vit. D3 1,8. $10^{6}$ UI; Vit. E 66,66 g; Vit. B1 6,66 g; Vit. B2 13,33 g; Pantotenato de Ca 33,33 g; Biotina 533,3 mg; Ac. Fólico 2,66 g; Ac. Ascórbico 400,0 g; Ac.

Nicotínico 100,0 g; Vit. $\$ 1220,0$ mg; Vit. K3 6,66 g; veículo q.s.p. $1,0 \mathrm{~kg}$

2 Premix microminerais â Lab. Roche S.A. : Composição por $100 \mathrm{~g}$ : Magnésio 1,0; Zinco 16,0; Ferro 4,0;

Cobre 1,0; lodo 0,5: Selênio 0,05; Cobalto 0,01. 
ram preparadas na forma de pellets secos com $5 \mathrm{~mm}$ de diâmetro e armazenadas em sacos plásticos.

Amostras dos resíduos, dos demais ingredientes das rações e da carcaça dos peixes de cada tratamento (início e final do experimento) foram trituradas, liofilizadas e homogeneizadas com a finalidade de determinar sua composição bromatológica. Estas análises foram feitas de acordo com a Association of Official Analitical Chemists - AOAC (1995) e foram determinados os seguintes compostos: umidade, proteína bruta, extrato etéreo, fibra bruta, cinza e carboidrato (Tab. 2).

A energia bruta (Kcal/100g) das amostras dos ingredientes e das rações foi obtida por meio de bomba calorimétrica modelo Parr 1271. Amostras com aproximadamente $1,0 \mathrm{~g}$ foram prensadas formando unidades de pastilhas, e colocadas para queimar na bomba. As correções para as substâncias formadas durante a combustão da amostra foram feitas utilizando-se titulação com solução de carbonato de sódio $(1 \mathrm{ml}$ da solução $=1 \mathrm{cal})$.

A energia bruta das amostras dos peixes (início e final do experimento) foi calculada de acordo com White et al. (1964), Halver (1972) e Jaramillo (1988), utilizando-se os seguintes fatores de conversão: $5,65 \mathrm{kcal} / \mathrm{g}, 4,15 \mathrm{kcal} / \mathrm{g}$ e $9,40 \mathrm{kcal} / \mathrm{g}$ para proteína, carboidratos e lipídios respectivamente.

Os parâmetros de desempenho dos peixes observados foram o ganho de peso (GP) e conversão alimentar aparente (CAA) e foram calculados para cada unidade experimental segundo Roubach (1991). A taxa de crescimento específico foi calculada segundo a seguinte equação: $\mathrm{TCE}=$ 100* ( $\mathrm{Ln}$ peso final - $\mathrm{Ln}$ peso inicial)/ período experimental em dias.

Com a finalidade de verificar a homogeneidade em peso dos peixes, ao início do experimento, foi aplicado o teste "F" a $5 \%$ de significância. Ao final do experimento os dados das biometrias de peso, do comprimento e da composição centesimal dos peixes foram analisados através do teste "F" a $5 \%$ de significância Banzatto \& Kronka (1989). O ganho de peso foi

Tabela 2. Composição centesimal aproximada das rações experimentais $(\mathrm{R} 1=21 \%$ de farinha de resíduo de peixe; $\mathrm{R} 2=14 \%$ de farinha de resíduo de peixe $+7 \%$ farinha de resíduo de frango; $\mathrm{R} 3=7 \%$ de farinha de resíduo de peixe $+14 \%$ farinha de resíduo de frango $\mathrm{R} 4=21 \%$ de farinha de resíduo de frango).

\begin{tabular}{cccccccc}
\hline Ração & MS & PB & EE & ENN & MM & FB & EB (Kcal/100g) \\
\hline R1 & 92,0 & 23,0 & 4,6 & 60,4 & 10,5 & 1,5 & 384,9 \\
R2 & 95,8 & 24,5 & 4,4 & 60,1 & 9,3 & 1,7 & 406,4 \\
R3 & 96,7 & 28,7 & 3,8 & 60,4 & 5,1 & 2,0 & 433,5 \\
R4 & 94,8 & 26,8 & 3,3 & 66,0 & 2,6 & 1,3 & 432,9 \\
\hline
\end{tabular}

$\mathrm{MS}=$ Matéria seca; $\mathrm{PB}=$ Proteina bruta; $\mathrm{EE}=$ Extrato etéreo; $\mathrm{ENN}=$ Extrato não nitrogenado; $\mathrm{MM}=\mathrm{Material}$ mineral; $\mathrm{FB}=$ Fibra bruta; $\mathrm{EB}=$ Energia bruta. 
analisado através de uma regressão quadrática com a finalidade de estabelecer, o ponto de inflexão da curva, onde ocorre o melhor desempenho (Mendes, 1999).

\section{RESULTADOS E DISCUSSÃO}

Os resultados das médias dos parâmetros de desempenho, da composição centesimal corporal e da energia bruta dos peixes no experimento, não apresentaram diferença significativa em nenhum dos tratamentos ( $p>0,05)$ (Tab. 3). Esses resultados indicam que todas às rações tem efeito semelhante sobre a qualidade corporal dos peixes e podem ser comparados aos valores encontrados por Roubach (1991) ao usar Pseudobombax munguba como fonte alternativa na alimentação do tambaqui, mas inferiores aos resultados do mesmo autor para o tratamento com ração balanceada que apresentou $60,9 \%$ de proteína bruta na carcaça dos peixes.

Conversões próximas da unidade são consideradas ótimas para o cultivo de peixes (Kubitza, 1998) mas nem sempre representam bons índices de crescimento. Merola \& Pagán-Font (1988) usando farinhas de peixe (10\%), de sangue $(5 \%)$, osso e $(5 \%)$ e de soja (25\%) como ingredientes de uma ração experimental obtiveram baixos índices de crescimento, $286,83 \mathrm{~g}$ em oito meses de cultivo de tambaqui, mesmo assim consideram que essa ração pode ser uma boa alternativa para o cultivo intensivo desse peixe devido à conversão alimentar aparente obtida que foi de 1,9. As conversões alimentares aparentes neste trabalho foram superiores a 2,0 em todos os tratamentos, e o melhor desempenho dos peixes pode ser observado nos tratamento R2 e R3 (Tab. 3).

Mesmo com conversões

Tabela 3. Médias obtidas ao final do período experimental de 120 dias para os parâmetros de desempenho e para a composição centesimal corporal (CCC) de juvenis de tambaqui, Colossoma macropomum, alimentados com rações a base de farinha de resíduo de peixe e de resíduo de frango. $(\mathrm{R} 1=21 \%$ de farinha de resíduo de peixe; $\mathrm{R} 2=14 \%$ de farinha de resíduo de peixe + $7 \%$ farinha de resíduo de frango; $\mathrm{R} 3=7 \%$ de farinha de resíduo de peixe $+14 \%$ farinha de resíduo de frango $\mathrm{R} 4=21 \%$ de farinha de resíduo de frango).

\begin{tabular}{ccccc}
\hline Parâmetro & $R 1$ & $R 2$ & $R 3$ & $R 4$ \\
\hline Peso final & $77,8 \pm 5,5^{\mathrm{a}}$ & $76,0 \pm 5,3^{\mathrm{a}}$ & $83,0 \pm 3,3^{\mathrm{a}}$ & $66,2 \pm 5,8^{\mathrm{a}}$ \\
CAA & 2,3 & 3,4 & 2,2 & 2,4 \\
Ganho de peso & $62,1 \pm 5,3^{\mathrm{a}}$ & $63,8 \pm 5,0^{\mathrm{a}}$ & $69,6 \pm 4,2^{\mathrm{a}}$ & $52,0 \pm 5,4^{\mathrm{a}}$ \\
TCE & $1,3 \pm 0,1$ & $1,5 \pm 0,2$ & $1,5 \pm 0,2$ & $1,2 \pm 0,1$ \\
\hline CCC (100\% MS) & $\mathrm{R} 1$ & $\mathrm{R} 3$ & $\mathrm{R} 4$ & $\mathrm{R} 2$ \\
\hline Umidade & $64,4 \pm 0,6^{\mathrm{a}}$ & $64,7 \pm 0,2^{\mathrm{a}}$ & $67,5 \pm 2,1^{\mathrm{a}}$ & $64,8 \pm 0,5^{\mathrm{a}}$ \\
PB & $45,6 \pm 0,8^{\mathrm{a}}$ & $44,08 \pm 0,4^{\mathrm{a}}$ & $45,58 \pm 0,6^{\mathrm{a}}$ & $45,65 \pm 1,0^{\mathrm{a}}$ \\
EE & $43,05 \pm 1,7^{\mathrm{a}}$ & $40,67 \pm 0,4^{\mathrm{a}}$ & $44,75 \pm 1,1^{\mathrm{a}}$ & $41,8 \pm 0,3^{\mathrm{a}}$ \\
Cinza & $10,6 \pm 0,3^{\mathrm{a}}$ & $9,97 \pm 0,6^{\mathrm{a}}$ & $9,4 \pm 0,2^{\mathrm{a}}$ & $8,5 \pm 0,4^{\mathrm{a}}$ \\
ENN & $2,3 \pm 1,4^{\mathrm{a}}$ & $5,2 \pm 0,7^{\mathrm{a}}$ & $0,8 \pm 0,2^{\mathrm{a}}$ & $4,0 \pm 1,2^{\mathrm{a}}$ \\
\hline Energia bruta & $667,1 \pm 9,7^{\mathrm{a}}$ & $653,0 \pm 3,5^{\mathrm{a}}$ & $679,7 \pm 5,63^{\mathrm{a}}$ & $667,8 \pm 3,5^{\mathrm{a}}$ \\
\hline
\end{tabular}

$\mathrm{CAA}=$ Conversão alimentar aparente; $\mathrm{TCE}=$ Taxa de crescimento especifico; $\mathrm{MS}=$ Matéria seca; $\mathrm{PB}=$ Proteina bruta; $\mathrm{EE}=$ extrato etéreo; $\mathrm{ENN}=\mathrm{Extrato}$ näo nitrogenado.

${ }^{a}=$ médias na mesma linha não diferem entre si estatisticamente (teste " $F "(p>0,05)$ ). 
alimentares semelhantes às de Ximenes-Carneiro (1991) para o tambaqui, o ganho de peso deste trabalho mostrou-se inferior ao encontrado por esse autor, que comparou duas rações na alimentação do tambaqui, com diferentes fontes de proteína: ensilado biológico e farinha de peixe e de carne, obtendo crescimento específico de 2,86 e 2,88 e conversões alimentares de 2,33 e 2,99 respectivamente. Essa diferença provavelmente esteja associada à qualidade nutricional dos ingredientes utilizados.

A qualidade da proteína é determinada pelo seu equilíbrio em aminoácidos (Kubitza, 1998) e influencia diretamente a qualidade nutricional da ração, que quando formulada adequadamente otimiza o desempenho do organismo que está sendo alimentado. Aminoácidos essenciais como metionina e lisina são limitantes, quando ausentes na alimentação, para o crescimento dos peixes (NRC, 1993). A farinha de resíduo de abate de aves do ponto de vista nutricional é um produto que rivaliza com farinhas de carne de boa qualidade devido ao nível aceitável de aminoácidos essenciais que possui (Andriguetto et al., 1986).

Os insumos usados neste trabalho para a fabricação da farinha de resíduo de peixe e de frango mostram um nível baixo de proteína bruta (Tab. 2), e podem ter influenciado para o baixo desempenho dos peixes. Melhores resultados para o desempenho do tambaqui foram encontrados por Merola \& Cantelmo (1987) utilizando $30 \%$ de proteína bruta na ração onde encontrou ganho de peso de $853 \mathrm{~g} \mathrm{em}$ 109 dias de cultivo.

A partir da Figura 1 pode-se observar que o melhor desempenho dos peixes ocorreu quando houve a combinação da farinha de resíduo de peixe e de frango. Estes resultados sugerem que esses ingredientes se complementam e melhoram o desempenho dos peixes.

Embora os resultados

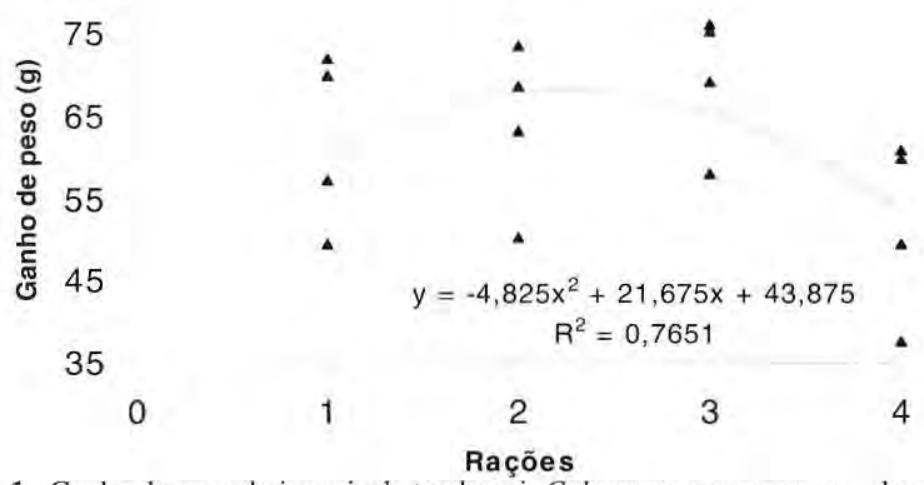

Figura 1. Ganho de peso de juvenis de tambaqui, Colossoma macropomum, durante um período experimental de 120 dias alimentados com ração experimental $(1=21 \%$ de farinha de resíduo de peixe; $2=14 \%$ de farinha de resíduo de peixe $+7 \%$ de farinha de resíduo de frango; $3=$ $7 \%$ de farinha de resíduo de peixe $+14 \%$ de farinha de resíduo de frango; $4=21 \%$ de farinha de resíduo de frango). 
encontrados para os parâmetros de desempenho neste trabalho sejam baixos, são encorajadores e sugerem a realização de um rearranjo desses ingredientes na elaboração de rações com a finalidade de torná-los uma boa alternativa para a alimentação do tambaqui.

\section{CONCLUSÕES}

Nas condições deste trabalho pode-se concluir que:

-As rações contendo farinha de resíduo de peixe e de frango possuem efeitos semelhantes sobre o desempenho dos juvenis de tambaqui Colossoma macropomum, mas a combinação desses ingredientes na ração proporcionam melhores resultados.

- A composição das rações, não afetou a composição corporal dos juvenis de tambaqui.

\section{AGRADECIMENTOS}

A empresa Varig agropecuária pelo fornecimento dos resíduos de frango, a Secretaria de Estado de Agricultura do Pará, por permitir o uso das instalações da estação de piscicultura de Santa Rosa, Santarém, ao Conselho Nacional de Pesquisas/ $\mathrm{CNPq}$ pelo suporte financeiro que possibilitou o desenvolvimento deste trabalho e a Bruno Adan Sagratzki Cavero pelas sugestões apresentadas.

\section{Bibliografia citada}

Andriguetto, J. M.; Gemael, A.; Souza, G. A.; Minardi, I.; Flemming, J.S.; Perly, L.; Flemming, R.; Vinne, L.U.V.D. 1986. Normas e padrões de nutrição $e$ alimentação animal - Revisão 85/86. Curitiba: Nutrição, 140p.

Andriguetto, J. M.; Perly, L.; Minardi, I.; Gemael, A.; Flemming, J.S.; Souza, G.A.; Bona-Filho, A. 1990. Nutrição Animal. $4^{a}$ ed. São Paulo: Nobel. 395p. v.1.

Association of Official Analytical Chemists. 1975. Official Methods of Analysis. $12^{\text {th }}$ Edition. George Banta Co. INC., Manasha, Wisconsin. 937p.

Banzatto, D.A.; Kronka, S.N. 1989. Experimentação Agrícola. Jaboticabal: FUNEP. 247p.

BASA. 1995. Análise setorial - Estudo sobre a pesca artesanal. Consultoria Técnica. Coordenação de Estudos Econômicos. Projeto Bra - 087/021 Pnud/Basa/Sudam/ Suframa. 55p. relatório mimeo.

Beaumariage, D.S. 1968. Manufature of fish meal from Florida's fishery waste and underexploited fishes; Technical Series, 54.

Falabella, P.R.G. 1994. A pesca no Amazonas: problemas e soluções. $2^{\mathrm{a}} \mathrm{ed}$. Manaus: Imprensa Oficial do Estado, 180p.

Freitas, J.V.F.; Gurgel, J.J.S. 1976. Sobre o aproveitamento de resíduos do pescado dos açudes do Nordeste, na elaboração de sub-produtos; Bol. Téc. DNOCS, v. 34, $n^{\circ}$ 2, p. 139-147.

FAO. 1990. Anuário Estadístico de la pesca mundial. Roma, 647p.

Graef, E.W. 1995. As espécies de peixes com potencial para criação no Amazonas. In: Val, A.L.; Honczaryk, A. (eds.) 1995. Criando peixes na Amazônia. Manaus: INPA, p. 29-43.

Halver, J.E. 1972. Fish nutrition. Academic Press. New York and London. 713p.

Jaramillo, N.D. 1988. Alimentación de peces. Requerimientos, cálculos de raciones, materias primas y dietas. Universidad de Caldas, Facultad de Medicina Veterinária y Zootécnia. Centro de Investigación Piscícola. Manizales. Colombia. 35p.

Junk, W.J. 1975. Ecology, fisheries and fish culture in Amazonia. In: The Amazon limnology and landscape ecology of a 
mightytropical river and its basin. Dr. W. Junk Publishers, Boston, p. 443-476.

Junk, W.J. 1979. Macrófitas aquáticas nas vârzeas da Amazônia e possibilidade de seu uso na agropecuária. INPA. Manaus, 24p. mimeo.

Kubitza, F., 1998. Nutrição e Alimentação dos Peixes Cultivados. Campo Grande. MS.

Mendes, P.P. 1999. Estatística aplicada à aqüicultura. Recife: Bagaço. 265p.: il.

Merola, N.; Cantelmo, O.A. 1987. Growth, feed conversion and mortality of cagereared tambaqui, Colossoma macropomum, fed various dietary feeding regimes and protein levels. Aquaculture, 66(3-4): 223-233.

Merola, N.; Pagan-Font. 1988. Pond culture of the amazon fish tambaqui, Colossoma macropomum, a pilot study. Aquacultural Engineering. 7: 113-125.

Merona, B.; Bittencourt, M.M. 1988. A pesca na Amazônia através dos desembarques no mercado de Manaus: resultados preliminares. Memória, Sociedade de Ciências Naturales la Salle, tomo XLVIII, suplemento, 433-453.

NRC. 1993. Nutrients requeriments of fish. National Research Council. Washington. D. C. $115 p$.

Pereira-Filho, M. 1982. Preparo e utilização de ingredientes produzidos em Manaus, no arraçoamento do matrinchã, Brycon sp. Instituto Nacional de Pesquisas da Amazônia. INPA/FUA. Manaus. Dissertação de Mestrado. 89p.

Petrere Jr., M. 1978. Pesca e esforço de pesca no Estado do Amazonas. II. Locais, aparelhos de captura e estatística de desembarque. Acta Amazonica, 8(3):1-54 suplem. 2.

Rodrigues, M.J.; Sakawaki, H.K.: Arana, H.N. C.; Silva, F.R.L. 1996. Aquicultura na Amazônia: o estado atual e perspectiva para o seu desenvolvimento. In: Políticas pesqueiras nos países amazônicos. Belém: UNAMAZ, UFPA/NAEA, 502p. (Série Cooperação Amazônica, 17). p.365-435.
Roubach, R. 1991. Uso de frutos e sementes de florestas inundáveis na alimentação de Colossoma macropomum (Cuvier 1818) (Pisces, Characidae). Dissertação de Mestrado. INPA/FUA, Manaus - AM, 79p.

Saint-Paul, U.1986. Potential for aquaculture of South American freshwater fishes: a review. Aquaculture, 54: 205-240.

Saint-Paul, U.; Werder, U. 1977. Aspectos genereales sobre la piscicultura en Amazonas y resultados preliminares de experimentos de alimentación com raciones peletizadas con diferentes composiciones. Simp. Asoc. Lat. Acuic., I. Maracay, Venezuela. 22p.

Saint-Paul, U.; Werder, U.; Teixeira, A.S. 1981. O uso do aguapé (Eichornia crassipes) em experimentos de alimentação de matrinxã (Brycon sp.) An. Simp. Bras. Aquic., II. Jaboticabal, São Paulo. p. 83-84.

Silva, J.A.M. 1997. Nutrientes, energia e digestibilidade aparente de frutos $e$ sementes consumidos pelo tambaqui (Colossoma macropomum CUVIER, 1818) nas florestas inundáveis da Amazônia Central. Tese de Doutorado. Manaus: INPA/FUA. 142p.

Torres, A.P. 1989. Alimentos e nutrição das aves domésticas. $2^{\mathrm{a}}$ ed. São Paulo: Nobel. 324p.

White, A.; Handler, P.; Smith, E.L. 1964. Principles of biochemistry. New York: McGraw-Hill. 1106p.

Ximenes-Carneiro, A.R. 1991. Elaboração e uso de ensilado biológico de pescado na alimentação de alevinos de tambaqui, Colossoma macropomum (Cuvier, 1818). Dissertação de Mestrado. INPA/FUA. Manaus-AM. 81p. 